NOTICE WARNING CONCERNING COPYRIGHT RESTRICTIONS:

The copyright law of the United States (title 17, U.S. Code) governs the making of photocopies or other reproductions of copyrighted material. Any copying of this document without permission of its author may be prohibited by law. 


\title{
Verifying Atomic Data Types
}

\author{
Jeannette M. Wing \\ 20 July 1989 \\ CMU-CS-89-168,
}

\author{
School of Computer Science \\ Carnegie Mellon University \\ Pittsburgh, PA 15213
}

To appear in the Proceedings of the REX Workshop on Stepwise Refinement of Distributed Systems: Models, Formalism, Correctness,

Plasmolen, The Netherlands, 1989

\begin{abstract}
Atomic transactions are a widely-accepted technique for organizing computation in fault-tolerant distributed systems. In most languages and systems based on transactions, atomicity is implemented through atomic objects, typed data objects that provide their own synchronization and recovery. Hence, atomicity is the key correctness condition required of a data type implementation. This paper presents a technique for verifying the correctness of implementations of atomic data types. The novel aspect of this technique is the extension of Hoare's abstraction function to map to a set of sequences of abstract operations, not just to a single abstract value. We give an example of a proof for an atomic queue implemented in a real programming language, Avalon/C++.
\end{abstract}

This research was sponsored by the Defense Advanced Research Projects Agency (DOD), ARPA Order No. 4976, monitored by the Air Force Avionics Laboratory Under Contract No. F33615-87-C-1499. Additional support was provided in part by the National Science Foundation under grant CCR-8620027.

The views and conclusions contained in this document are those of the author and should not be interpreted as representing the official policies, either expressed or implied, of the Defense Advanced Research Projects Agency, the National Science Foundation or the U.S. Government. 
1. Introduction

\section{Table of Contents}

2. Model for Transaction-Based Distributed Systems

2.1. Histories

2.2. Legality of Sequential Histories

2.3. Atomicity $=$ Serializability + Recoverability

2.3.1. Local Atomicity

2.3.2. On-line Atomicity

3. Verification Method

4. Implementing Atomlc Objects

4.1. Transaction Identiflers

4.2. Ensuring Serlalizability and Recoverability

5. An Example: A Highly Concurrent FIFO Queue

5.1. The Implementation

5.1.1. The Representation

5.1.2. The Operations

5.2. Application of Verification Method

5.2.1. Representation Invariant

5.2.2. Abstraction Function

5.2.3. Type-Speciflc Correctness Condition

5.3. Verlfying the Implementation

5.3.1. Proof Sketch

5.3.2. Formal Proof for Enqueue and Dequeue

6. Discussion and Related Work

6.1. Hybrid Atomicity Revisited

6.2. Abstraction Functions Revisited

6.3. Other Models for Transactions

7. Current and Future Work

8. Acknowledgments

References 


\section{List of Figures}

Figure 2-1: Interfaces for Queue Operations $\quad 5$

Figure 2-2: Tralt for Queue Values $\quad 5$

Figure 5-1: An Example of Why an Enqueuer (B) Must Walt $\quad 16$

Flgure 5-2: An Example of Why a Dequeuer (B) Must Walt $\quad 16$

Figure 5-3: An Example of When an Enqueuer (C) Need Not Wait 17

Figure 5-4: An Example Queue Representation State 18

Figure 6-1: Relationships Among Atomiclty Properties 25 


\section{Introduction}

A distributed system consists of multiple computers (called nodes) that communicate through a network. Programs written for distributed systems, such as airline reservations, electronic banking, or process control, must be designed to cope with failures and concurrency. Concurrency arises because each rocess executes simultaneously with other processes on the local node and processes on remote nodes, while failures arise because distributed systems consist of many independently-failing components. Typical failures include node crashes, network partitions, and lost messages.

A widely-accepted technique for preserving consistency in the presence of failures and concurrency is to organize computations as sequential processes called transactions. Transactions are atomic, that is, serializable and recoverable. Informally, serializability [32] means that concurrent transactions appear to execute sequentially, and recoverability means that a transaction either succeeds completely or has no effect. A transaction's effects become permanent when it commits, its effects are discarded if it aborts, and a transaction that has neither committed or aborted is active.

In most languages and systems based on transactions, atomicity is implemented through atomic objects, which are typed data objects that provide their own synchronization and recovery. Languages such as Argus [24], Avalon [18], and Aeolus [39] provide a collection of primitive atomic data types, together with constructs allowing programmers to define their own atomic types. The most straightforward way to define a new atomic type is to use an existing atomic data type as a representation, but objects constructed in this way often support inadequate levels of concurrency [37]. Instead, one could implement new atomic objects by carefully combining atomic and non-atomic components and exploiting the semantics of the data type to provide more encurrency. This degree of freedom comes with a price: the programmer is now responsible for proving that the implementation of the user-defined data type is indeed atomic.

In this paper, we formulate proof techniques that allow programmers to verity the correctness of atomic objects. Although language and system constructs for implementing atomic objects have received considerable attention in the distributed systems community, the problem of vertfying the correctness of programs that use those constructs has received surprisingly little attention. To our knowledge, the Avalon Project conducted at Carnegie Mellon University is the only language project to address this particular program verification problem.

Tectiniques for reasoning about concurrent programs are well-known [2, 19, 22, 31], but are not adequate for reasoning about atomicity. They typically address issues such as mutual exclusion or the atomicity of individual operations; they do not address the more difficult probterns of ensuring the serializability of arbitrary sequences of operations, nor do they address recoverability. Reasoning about atomicity is inherently more difficult than reasoning about concurrency alone.

Our work distinguishes us from most other formal specification and verification research in concurrent and distributed systems since we address the presence of failures as seriously as the presence of concurrency and distribution. Our particular approach also distinguishes our work from many others: we 
focus on the behavior and correctness of objects in a system and not on the processes (transactions) that manipulate them. We base the proof of correctness of the entire system on a local property of the objects in the system; if the property holds for each object, the correctness of the entire system is guaranteed. Thus, we transform the problem of proving an entire distributed system correct into the more manageable problem of proving each of the objects in the system correct.

This paper is organized as follows. In Section 2 we present our model and basic definitions, and illustrate most of them through simple examples. In Section 3 we describe three pieces in our verification technique, the most important of which is an extension of Hoare's abstraction function for data implementations. In Section 4, we introduce and motivate relevant Avalon/C++ programming language primitives. We give in Section 5 an extended example using these primitives and a correctness proof following the technique outtined in Section 3. Section 6 discusses related work, in particular contrasting the particular correctness condition we use with another more conventional one and contrasting our extended abstraction function with other kinds of mappings. Finally, we close in Section 7 with a summary of relevant current and future work.

\section{Model for Transaction-Based Distributed Systems}

A distributed system is composed of a set of transactions and a set of objects. A transaction corresponds to a sequential process. We disallow concurrency within a transaction, but allow for multiple transactions to execute concurrently. Objects contain the state of the system. Each object has a type, which defines a set of possible values and a set of operations that provide the only means to create and manipulate objects of that type. A transaction can either complete successfully, in which case it commits, or unsuccessfully, in which case it aborts. We use the term termination for the end of the execution of an operation and completion for the end of the execution of a transaction.

Typically, a transaction executes by invoking an operation on an object, receiving results when the operation terminates, then invoking another operation on a possibly different object, receiving results when it terminates, etc. It then commits or aborts.

Although Avalon permits transactions to be nested [29, 33], the model presented here and our subsequent discussion consider only single-level transactions. Nested transactions provide a means to obtain concurrency within a transaction; Lynch and Merritt [26] present a formal model of nested transactions based on 1/O automata. Our model of transactions borrows heavily from Weihl's, first described in his 1984 Ph.D. thesis [36] and more recently, in [38].

\subsection{Historles}

We model a computation as a history, which is a finite sequence of events. There are four kinds of events: invocations, responses, commits, and aborts. An invocation event is written as $x$ op(args") $A_{1}$ where $x$ is an object name, op an operation name, args" a sequence of arguments, and $A$ a transaction name. A response event is written as $x$ term(res*) A, where term is a termination condition, and res ${ }^{*}$ is a 
sequence of results. We use "Ok" for nomal termination. A commit or abort event is written $x$ Commit $A$ or $x$ Abort $A$, and it indicates that the object $x$ has learned that transaction $A$ has committed or aborted.

A response matches an earlier invocation if their object names agree and their transaction names agree. An invocation is pending if it has no matching response. An operation in a history is a pair consisting of matching invocation and response events. An operation $o p_{0}$ lies within $o p_{1}$ in $H$ if the invocation event for $o p_{1}$ precedes that of $o p_{o}$ in $H$, and the response event for $o p$, follows that of $o p_{o}$. For histories, we use "•" to denote concatenation, and " $\Lambda$ " the empty history.

For a history $H$, we define committed(H) to be the set of transactions in $H$ that commit in $H$, and aborted $(H)$ to be the set of transactions that abort in $H$. We define completed(H) to be committed $(H) \cup$ aborted $(H)$, and active $(H)$ to be the set of transactions in $H$ not in completed(H). Note that we can model a failure event (e.g.. node crash) with abort events.

\section{Example}

The following history, $H_{1}$, involves two queue objects $p$ and $q$, and four transactions $A, B, C$, and $D$ :

$\begin{array}{lll}\text { p } & \text { Enq(1) } & \text { A } \\ \text { p } & \text { Enq(2) } & \text { B } \\ \text { p } & \text { Ok() } & \text { B } \\ \text { q } & \text { Enq(4) } & \text { B } \\ \text { p } & \text { Ok() } & \text { A } \\ \text { q } & \text { Ok() } & \text { B } \\ \text { p } & \text { Commit } & \text { B } \\ \text { q } & \text { Commit } & \text { B } \\ \text { p } & \text { Enq(3) } & \text { A } \\ \text { p } & \text { Ok() } & \text { A } \\ \text { p } & \text { Abort } & \text { A } \\ \text { p } & \operatorname{Deq}() & \text { C } \\ \text { p } & \text { Ok(2) } & \text { C } \\ \text { q } & \text { Enq(5) } & \text { D } \\ \text { p } & \text { Enq(6) } & \text { C } \\ \text { p } & \text { Ok() } & \text { C }\end{array}$

The first event in $H_{1}$ is the invocation of the Enq operation on object $p$ by transaction $A$. The fitth event is the matching response event. The seventh and eighth events indicate that $p$ and $q$ respectively have leamed that $B$ has committed; the eleventh indicates that $p$ has learned that $A$ has aborted. The Enq operation of 2 by $B$ lies within the Enq of 1 by $A$.

$A$ and $B$ execute concurrently and both eventually complete, $A$ unsucessfully and $B$ successfully. $C$ and $D$ execute concurrently and are both active (have neither committed nor aborted) at the end of $H_{1}$. Hence, committed $\left(H_{1}\right)=\{B\}$, aborted $\left(H_{1}\right)=\{A\}, \operatorname{completed}\left(H_{1}\right)=\{A, B\}$, and active $\left(H_{1}\right)=\{C, D\}$. When $C$ dequeves trom $p$, it recoives a 2. DS invocation of Enq on $q$ is pending since there is no matching response event. 


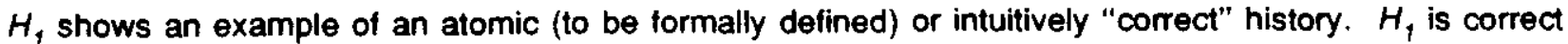
because there is some ordering on nonaborted transactions that is "equivalent" to a "sequential" version of $H_{1}$ and because $A$ 's effects are ignored. It would have been incorrect for $C$ to dequeue 1 from $p$ since $A$ aborts. If $A$ were to commit instead, then it would be correct either to have $C$ dequeue 1 , by ordering $A$ before $B$, or to have $C$ dequeue a 2 , by ordering $B$ before $A$. Notice that a transaction can perform more than one operation, possibly on different objects. A performs two Enq's on $p$ and $B$ performs one each on $p$ and $q$. The intuition we would like to capture in our formal definitions is as follows: At the end of $H_{1}$ (1) $p$ 's first and only element is either 2 ( $C$ aborts) or 6 ( $C$ commits); and (2) q's first and only element is 4 ( $q$ does not have 5 in it because $D^{\prime} s$ invocation is pending, yet it definitely has a 4 in it because $B$ s commit precedes $D$ s invocation).

\section{End example}

A transaction subhistory, $H \mid A(H$ at $A)$, of a history $H$ is the subsequence of events in $H$ whose transaction names are $A$. $H \mid S$ and $H \mid x$ are defined similarly, where $S$ is a set of transactions and $x$ is an object. Informally, two histories $H$ and $G$ are equivalent if for each transaction $A$, ignoring pending invocations, A performs the same events in the same order in $H$ as in $G$.

Definition 1: Let terminated $(H)$ denote the longest subhistory of $H$ such that every invocation has a matching response. Histories $H$ and $G$ are equivalent if terminated(H)|A= terminated(G) $\mid$ A for all transactions $A$.

If $H$ and $G$ are equivalent, then for all objects $x$ the state of $x$ after $H$ should be the same as that of $x$ after $G$; the converse is not true.

Definition 2: A history , well-formed if it satisfies the following conditions for all transactions A:

1. The first event of $H \mid A$ is an invocation.

2. Each invocation in $H \mid A$, except possibly the last, is immediately followed by a matching response or by an abort event.

3. Each response in $H \mid A$ is immediately preceded by a matching invocation, or by an abort event.

4. If $H \mid A$ includes a commit event, no invocation or response event may follow it.

5. A transaction can either commit or abort, but not both, i.e., committed(H) $\cap$ aborted(H) $=\varnothing$.

These constraints capture the requirement that each transaction performs a sequence of operations. It cannot invoke one operation on an object $x$ and then another on $x$ (or any other object) without first receiving a response from its first invocation. If a transaction commits, it cannot have any pending invocations; If it aborts, it may be in the middle of executing an operation, and thus have at most one pending invocation. Once a transaction commits, it cannot perform turther operations.

Definition 3: A well-formed history $H$ is sequential if:

1. Transactions are not interleaved. That is, if any event of transaction $A$ precedes any event of $B$, then all events of $A$ precede all events of $B$.

2. All transactions, except possibly the last, have committed. 


\section{Examples}

$H_{1}$ is well-formed. $H_{1} \mid B$ is the transaction subhistory:

$$
\begin{array}{lll}
\text { p } & \text { Enq(2) } & \text { B } \\
\text { p } & \text { Ok() } & \text { B } \\
\text { q } & \text { Enq(4) } & \text { B } \\
\text { q } & \text { Ok() } & \text { B } \\
\text { p } & \text { Commit } & \text { B } \\
\text { q } & \text { Commit } & \text { B }
\end{array}
$$

and $H_{1} \mid p$ is the object subhistory:

$\begin{array}{lll}\text { p } & \text { Enq(1) } & \text { A } \\ \text { p } & \text { Enq(2) } & \text { B } \\ p & \text { Ok() } & \text { B } \\ \text { p } & \text { Ok() } & \text { A } \\ \text { p } & \text { Commit } & \text { B } \\ \text { p } & \text { Enq(3) } & \text { A } \\ \text { p } & \text { Ok() } & \text { A } \\ \text { p } & \text { Abort } & \text { A } \\ \text { p } & \text { Deq() } & \text { C } \\ \text { p } & \text { Ok(2) } & \text { C } \\ \text { p } & \text { Enq(6) } & \text { C } \\ \text { p } & \text { Ok() } & \text { C }\end{array}$

The following well-formed subhistory of $H_{1}$ is sequential:

$\begin{array}{lll}\text { p } & \text { Enq(2) } & \text { B } \\ \text { p } & \text { Ok() } & \text { B } \\ \text { q } & \text { Enq(4) } & \text { B } \\ \text { q } & \text { Ok() } & \text { B } \\ \text { p } & \text { Commit } & \text { B } \\ \text { q } & \text { Commit } & \text { B } \\ \text { p } & \text { Deq() } & \text { C } \\ \text { p } & \text { Ok(2) } & \text { C } \\ \text { p } & \text { Enq(6) } & \text { C } \\ \text { p } & \text { Ok() } & \text { C }\end{array}$

End examples

\subsection{Legality of Sequential Historles}

Each object has a sequential specification that defines a set of legal sequential histories for that object. To be concrete in this paper, we use the Larch specification approach [16] to write sequential specifications for objects. Other axiomatic approaches (e.g., lota [30], Clear [7], or OBJ [14]), or other specification methods, such as operational (e.g., VDM [6]) or state-machine oriented (e.g., vO automata [27]) methods, would be just as appropriate. 
the Enq and Deq operations for FIFO sequential queues are shown in Figure 2-1. A requires clause states the precondition that must hold when an operation is invoked. An ensures clause states the postcondition that the operation must establish upon termination. An unprimed argument formal, e.g., q, in a predicate stands for the value of the object in which the operation begins. A return formal or a primed argument formal, e.g., q', stands for the value of the object at the end of the operation. The specification for Deq is partial since Deq is undefined for the empty queue.

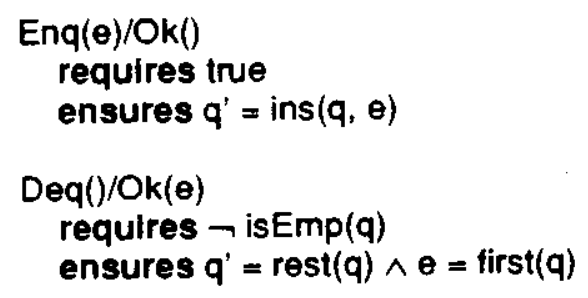

Figure 2-1: Interfaces for Queue Operations

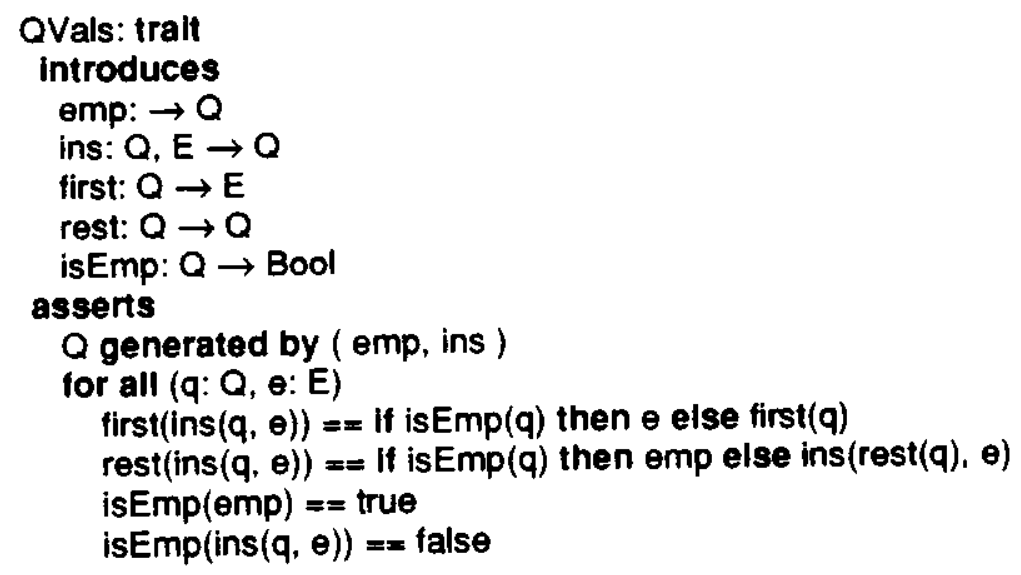

Figure 2-2: Trait for Queue Values

The assertion language for the pre- and postconditions is based on traits written in the Larch Shared Language as in Figure 2-2. A trait is akin to an algebraic specification and is used to describe the set of values of a typed object. The set of operators and their signatures following introduces defines a vocabulary of terms to denote values. For example, emp and ins(emp, 5) denote two different queve values. The set of equations following the asserts clause defines a meaning for the terms, more precisely, an equivalence relation on the terms, and hence on the values they denote. For example, from QVals, we could prove that rest(ins(ins(emp, 3), 5))) $=$ ins(emp, 5). The generated by clause of QVals asserts that emp and ins are sufficient operators to generate all values of queues. Formally, it introduces an inductive rule of inference that allows one to prove properties of all terms of sort $Q$. We use the vocabulary of traits to write the assertions in the pre- and postconditions of a type's operations; we use the meaning of equality to reason about its values.

Definition 4: Given a sequential specification of an object, a sequential object history is legal if the state of the object before each invocation event satisfles the pre-condition of the object's 
invoked operation and the state of the object before each matching response satisfies the corresponding postcondition.

A sequential history $H$ involving multiple objects is legal if it is legal at each object, i.e., each subhistory $H \mid x$ is legal with respect to the sequential specification for $x$.

\subsection{Atomicity $=$ Serlalizability + Recoverability}

We are interested in defining when a history is atomic, i.e., serializable and recoverable. We first define when a history is serializable and then when it is atomic, by adding the recoverability property.

Definition 5: If $H$ is a history and $T$ is a total order on transactions, $\operatorname{Seq}(H, T)$ is the sequential history equivalent to $H$ in which transactions appear in the order $T$.

For example, if $A_{1}, \ldots, A_{n}$ are transactions in $H$ in the order $T$, then $\operatorname{Seq}(H, T)=H\left|A_{1} \bullet \ldots \bullet H\right| A_{n}$.

Serializability picks off only those equivalent sequential histories that are legal.

Definition 6: Let $S=$ committed $(H) \cup$ active $(H)$ in a history $H . H$ is serializable if there exists

some total order $T$ on the transactions in $S$ such that $S e q(H / S, T)$ is legal.

$S$ is the set of transactions in $H$ that have committed or are still active, and thus, does not include aborted transactions. Unrolling the above two definitions, serializability requires only that we find some total order $T$ on nonaborted transactions in $H$ that yields a legal sequential equivalent history.

\section{Example}

$H_{1}$ is serializable because ordering the transaction $B$ before $C$ is equivalent to the sequential history,

$\begin{array}{lll}\text { p } & \text { Enq(2) } & \text { B } \\ \text { p } & \text { Ok() } & \text { B } \\ \text { q } & \text { Enq(4) } & \text { B } \\ \text { q } & \text { Ok() } & \text { B } \\ \text { p } & \text { Commit } & \text { B } \\ \text { q } & \text { Commit } & \text { B } \\ \text { p } & \text { Deq() } & \text { C } \\ \text { p } & \text { Ok(2) } & \text { C } \\ \text { p } & \text { Enq(6) } & \text { C } \\ \text { p } & \text { Ok() } & \text { C }\end{array}$

which is legal because $C$ correctly dequeues 2, placed at the head of the queve by $B$. Notice that "equivalence" lets us ignore $D$ because it has only a pending invocation in $H_{1}$ and "serializable" lets us ignore $A$ because it aborts in $H_{r}$. Thus, we need only order $B$ and $C$.

\section{End example}

Atomicity requires not only serializability, but recoverability as well. To define when a history is atomic, we simply restrict $S$ to be just the set of committed transactions in $H$.

Definition 7: $H$ is atomic if $H \mid$ committed $(H)$ is serializable.

Recoverability lets us ignore noncommmitted (i.e., aborted and active) transactions; we require that the resulting history be serializable. $H_{1}$ is atomic because it is equivalent to the sequential history that 
contains just the events of the one committed transaction $(B)$ in $H_{y}$.

\subsubsection{Local Atomicity}

The only practical way to ensure atomicity in a decentralized distributed system is to have each object perform its own synchronization and recovery. In other words, we want to be able to verify the atomicity of a system composed of multiple objects by verifying the atomicity of individual objects.

However, atomicity as defined so far is too weak a property to let us perform such local reasoning. That is, $H$ is not necessarily atomic just because $H \mid x$ is atomic for each object $x$. For example, suppose $s$ and $t$ are set objects. The following history $H_{2}$ is not atomic, even though $H_{2} \mid s$ and $H_{2} \mid t$ both are:

$\begin{array}{lll}s & \operatorname{lns}(1) & A \\ s & O k() & A \\ t & M e m(2) & A \\ t & O k(t r u e) & A \\ s & M e m(1) & B \\ s & O k(t r u \theta) & B \\ t & \operatorname{lns}(2) & B \\ t & O k() & B \\ s & C o m m i t & A \\ s & \text { Commit } & B \\ t & \text { Commit } & A \\ t & \text { Commit } & B\end{array}$

$\mathrm{H}_{2} \mid \mathrm{s}$ is serializable in the order in which $A$ precedes $B$ and $H_{2} \mid t$ is serializable in the order in which $B$ precedes $A$, but $\mathrm{H}_{2}$ clearly cannot be serializable in an order consistent with both.

To ensure that all objects choose compatible serialization orders, it is necessary to impose certain additional restrictions on the behavior of atomic objects. These restrictions let us reason about atomicity locally. Thus, if each object is guaranteed to satisfy a local atomicity property, the entire system will be globally atomic. Avalon/C++ uses a local atomicity property that Weihl calls hybrid atomicity [36]. Informally, a history $H$ is hybrid atomic if it is serializable in the order in which the transactions in $H$ commit.

To capture formally the restriction that transactions must be serializable in commit-time order, we make the following adjustments to our model. When a transaction commits, it is assigned a logical timestamp [21], which appears as an argument to that transaction's commit events. These timestamps determine the transactions' serialization order. Commit timestamps are subject to the following well-formedness constraint, which reflects the behavior of logical clocks: if $B$ executes a response event after $A$ commits, then $B$ must receive a later commit timestamp. For a given history $H$, let $T S(H)$ be the partial order such that $(A, B) \in T S(H)$ if $A$ and $B$ commit in $H$ and the timestamp for $A$ is less than the timestamp for $B$. $T S(H)$ defines a total order on committed(H).

Definition 8: A history $H$ is hybrid atomic if $H \mid$ committed $(H)$ is serializable in the order $T S(H)$.

Serializability requires only that there exists some total order on transactions in $H$, atomicity implies we 
neөd order only the committed transactions; finally, hybrid atomicity picks an order (commit-time order) for which there must be a legal sequential equivalent. Weihl shows that hybrid atomicity is an optimal local atomicity property: no strictly weaker local property sutfices to ensure global atomicity [36].

Objects may learn of the commitment of transactions in an order different from the actual commit-time order. This behavior reflects real distributed systems where long delays or unreliable transmission of messages may cause objects not to have the most up-to-date view of the entire system. An object may not know that a committed transaction $A$ has committed, and hence believe $A$ is still active. The following history,

$\begin{array}{lll}\text { s } & \operatorname{lns}(1) & \text { A } \\ \text { s } & \operatorname{Ins}(2) & \text { B } \\ \text { s Ok() } & \text { B } \\ \text { s Ok() } & \text { A } \\ \text { s } M e m(2) & \text { A } \\ \text { s Ok(false) } & \text { A } \\ \text { s Commit(1:15) } & \text { B } \\ \text { s Commit(1:00) } & \text { A }\end{array}$

is hybrid atomic since it is serializable in the order in which $A$ precedes $B$. Here, $s$ leams about the commitment of $A$ after it learns about the commitment of $B$, even though $A$ commits before $B$.

Though all hybrid atomic histories are atomic, not all atomic histories are hybrid atomic [36]. Ignoring the timestamp arguments to the commit events, the following history,

$\begin{array}{ll}\text { s } \operatorname{lns}(1) & \text { A } \\ \text { s } O k() & \text { A } \\ \text { s } M e m(1) & \text { B } \\ \text { s Ok(false) } & \text { B } \\ \text { s Commit (1:00) } & \text { A } \\ \text { s Commit(1:15) } & \text { B }\end{array}$

is atomic, but not hybrid atomic. It is serializable in the order in which $B$ precedes $A$, but not in which $A$ precodes $B$.

Since hybrid atomicity is local, we henceforth need only consider object subhistories.

\subsubsection{On-line Atomiclty}

Since an object may hear about the commitment of transactions out-of-order, it may be difficult for it to choose an appropriate response to a pending invocation of an active transaction. Thus, we focus on "pessimistic" atomictty, where an active transaction with no pending invocation is always allowed to commit. Using this stronger property, called on-line hybrid atomicity, gives us the additional advantage that we can perform inductive reasoning over events in a history, which is not possible using simple hybrid atomicity.

Definttion 9: $H$ is on-line atomic if every well-formed history $H$ 'constructed by appending wellformed commit events to $H$ is atomic. We call any sequential history equivalent to $H^{\prime} \mid$ committed( $\left.H^{\prime}\right)$ a serialization of $H$. 
This definition implies that $H$ is on-line atomic if every one of its serializ ations is legal. We will typically work with serializations of $H$, letting us tack on zero, one, or more commit events to $H$. On-line atomicity allows us to choose to complete any number of active transactions, and thereby introduces inherent nondeterminism into our correctness condition.

\section{Examples}

The following history,

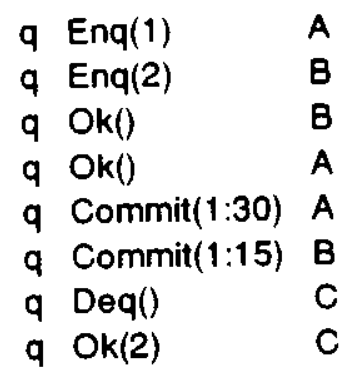

is on-line (hybrid) atomic. It has two serializations: on $\theta$ in which $B$ precedes $A$, and one in which $B$ precedes $A$ and $A$ precedes $C$, and it is easily verified that both are legal.

However, the following history, $H_{3}$

$\begin{array}{lll}q & \text { Enq(1) } & \text { A } \\ q & \text { Enq(2) } & \text { B } \\ q & \text { Ok() } & \text { B } \\ q & \text { Ok() } & \text { A } \\ q \text { Commit(1:15) } & \text { B } \\ q & \text { Deq() } & \text { C } \\ q & \text { Ok(2) } & \text { C }\end{array}$

is hybrid atomic but not on-line hybrid atomic, since the history $H_{3}^{\prime}=H_{3} \bullet q$ Commit(1:00) $A \bullet$ $q$ Commit $(1: 30) C$ is not serializable in the order in which $A$ precedes $C$.

\section{End examples}

In summary, we henceforth consider a history to be atomic if its transactions are serializable in committime order, and to be on-line atomic if the result of appending commit events with well-formed commit timestamps is atomic.

\section{Verification Method}

We first define our notion of correctness based on the atomicity property presented in the previous section. We then give a verification method for proving the correctness of implementations of atomic objects.

An implementation is a set of histories in which events of two objects, a representation object $r$ of type Rep and an abstract object $a$ of type Abs, are interleaved in a constrained way: for each history $H$ in the 
implementation, (1) the subhistories $H \mid r$ and $H \mid$ a satisfy the usual well-formedness conditions; and (2) for each transaction $A$, each representation operation in $H \mid A$ lies within an abstract operation. Informally, an abstract operation is implemented by the sequence of representation operations that occur within it.

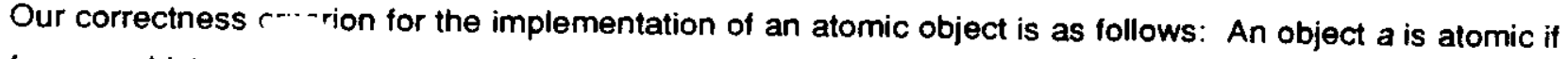
for every history implementation, $H \mid a$ is atomic. We typically do not require $H \mid r$ to be atomic.

To show the correctness of an atomic object implementation, we must generalize techniques from the sequential domain. We use three "tools" in our method: (1) a representation invariant, (2) an abstraction function, and (3) the object's sequential specification. The representation invariant defines the domain of the abstraction function. The abstraction function maps a representation value to a set of sequences of abstract operations. The sequential specification determines which of those sequences are legal. The only unusual aspect of any of these tools is the range of the abstraction function: it is not a set of abstract values, but a powerset of sequences of abstract operations.

Let $R e p$ be the implementation object's set of values, $A b s$ be the set of values of the (sequential) data type being implemented, and $O P$ be the sequential object's set of operations. The subset of Rep values that are legal values is characterized by a predicate called the representation invariant, $t:$ Rep $\rightarrow$ bool. The meaning of a legal representation is given by an abstraction function, $A: R e p \rightarrow 2^{O P^{*}}$, defined only for values that satisfy the invariant. Unlike Hoare's abstraction functions for sequential objects [20] that map a representation value to a single abstract value, our abstraction functions map a representation value to a set of sequential histories of abstract operations.

Our basic verification method is to show inductively over events in a history that the following properties are invariant. Let $r$ be the representation state of the abstract object a after accepting the history $H$, and let $\operatorname{Ser}(H)$ denote the set of serializations of $H / a$.

1. $\forall S \in A(r), S$ is a legal sequential history, and

2. $\operatorname{Ser}(H) \subseteq A(r)$.

These two properties ensure that every serialization of $H$ is a legal sequential history, and hence that $H$ is on-line atomic. We use the object's sequential specification to help establish the first property. Note that if we were to replace the second property with the stronger requirement that $S e r(H)=A(r)$, then we could not verify centain correct implementations that keep track of equivalence classes of serializations. In the inductive step of our proof technique, we show the invariance of these two properties across a history's events, e.g., as encoded as statements in program text.

\section{Implementing Atomic Objects}

Given that atomicity is the tundamental correctness condition for objects in a transaction-based distributed system, how does one actually implement atomic objects? In this section we discuss some of the programming language support needed for constructing atomic objects. We have bullt this support in a programming language called Avalon/C++ [8], which is a set of extensions to $C_{++}$[35]. 
Essentially, Avalon/C++ provides ways to enable programmers to define abstract atomic types. For example, if we want to define an atomic_array type, we define a new class, atomic_array, which perhaps provides fetch and store operations. (Syntactically, a class is a collection of members, which are the components of the object's representation, and a collection of operation implementations.) The intuitive difference between a conventional array type and an atomic_array type is that objects of array type will not in general ensure serializability and recoverability of the transactions that access them whereas objects of atomic_array type will. However, the programmer who defines the abstract atomic type is still responsible for proving that the new type is correct, i.e., that all objects of the newly defined type are atomic. By providing language support for constructing atomic objects, we gain the advantage that this proof is done only once per class definition, not each time a new object is created. The verification method used for proving that an atomic type definition is correct is the heart of this paper.

Avalon/C++ has two built-in classes that together let programmers build atomic objects. The trans_id class provides operations that let programmers test the serialization order (i.e., commit-time order) of transactions at runtime. The subatomic class provides operations that let programmers ensure transaction serializability and recoverability.

\subsection{Transactlon Identifiers}

The Avalon/C++ trans_id (transaction identifier) class provides ways for an object to determine the status of transactions at runtime, and thus synchronize the transactions that attempt to access it. Trans_id's are a partially ordered set of unique values. Here is the trans_id class definition:

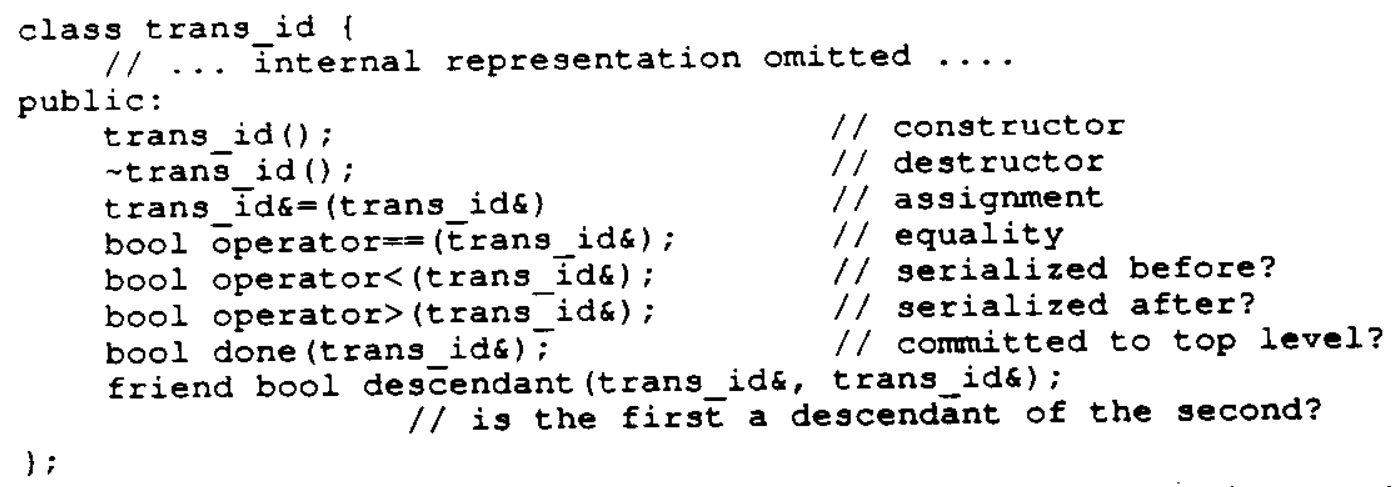

The three operations provided by trans_id's relevant to this paper are the creation operation, the comparison operation, and the descendant predicate.

The creation operation, called as follows:

trans_id $t=$ trans_id();

creates a new dummy subtransaction, commits it, and returns the subtransaction's trans_id to the parent transaction. Each call to the creation operation is guaranteed to retum a unique trans_id. A trans_id is typically used as a tag on an operation. Calling the trans_id constructor allows a transaction to generate multiple trans_id's ordered in the serialization order of the operations that created them. 
The comparison operation, used in the following expression, $t 1<t 2$

returns information about the order in which its arguments were created. If the comparison evaluates to true, then (1) every serialization that includes the creation of $t 2$ will also include the creation of $t 1$, and (2) the creation of $t 1$ precedes the creation of $t 2$. If $t 1$ and $t 2$ were created by distinct transactions $T 1$ and $T 2$, then a successful comparison implies that $T 1$ is committed and serialized before $T 2$, while if $t 1$ and $t 2$ were created by the same transaction, then $t 1$ was created first. If the comparison evaluates to false, then the trans_id's may have the reverse ordering, or their ordering may be unknown.

Comparison induces a partial order on trans_id's that "strengthens" over time: if $t 1$ and $t 2$ are created by concurrent active transactions, they will remain incomparable until one or more of their creators commits. If a transaction aborts, its trans_id's will not become comparable to any new trans_id's. Hence, "<" is capturing the commit-time order, i.e., serialization order, for committed transactions.

Finally, we use the descendant operation to compare whether a trans_id $t^{\prime}$ is a child of another. If the expression

descendant $\left(t^{\prime}, t\right)$

evaluates to true, then $t^{\prime}$ was created by the transaction $t$. Typically $t$ is the $t r a n s$ id of a committing or aborting transaction; the predicate lets us identity all its children.

Avalon/C++ maintains a logically global trans_id tree that provides the information on the relationship among trans_id's and the status of each transaction associated with a trans_id.

\subsection{Ensuring Serializabllity and Recoverability}

An atomic object in Avalon is defined by a $C_{++}$class that inherits from the Avalon buitt-in class subatomic. Here is the subatomic class definition:

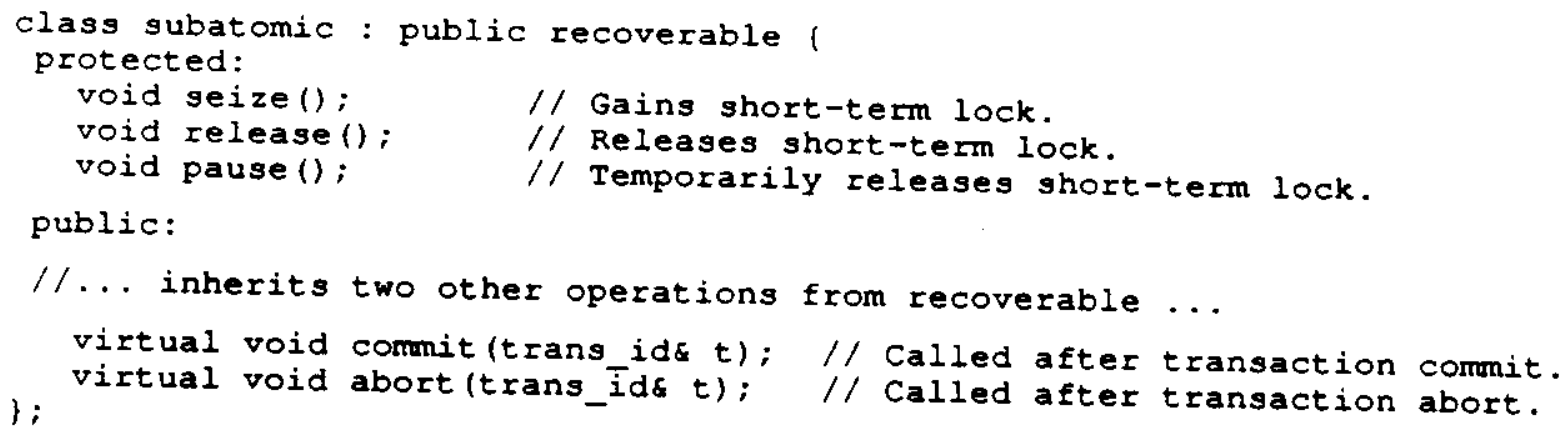

A programmer defining a new atomic data type derives from class subatomic, gaining access to all the above operations. The details of each of these operations are not important to this paper. Roughly speaking, the first three operations permit the implementation of each of the operations of the userdefined atomic data type to be executed "indivisibly." This property is conventionally called "atomic," where atomicity is at the level of an individual operation (i.e., "all-or-nothing" of a single operation), as opposed to atomicity at the level of a transaction (i.e., "all-or-nothing" of a sequence of operations). The 
last two operations allow implementors control over the clean-up processing done by an object when it leams that a transaction has committed or aborted.

With occasional minor variations, the implementation of each operation, op, of an atomic data type, atomic_T, which inherits from class subatomic, has the following form:

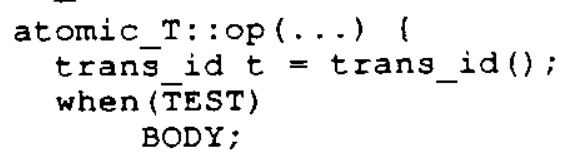

As previously explained, the call to the creation operation of trans_id generates a new trans_id which is used to "tag" the current call to op. The when statement is a conditional critical region: BODY is executed only when TEST evaluates to true. Avalon/C++ implements the when statement in terms of the seize, release, and pause operations of subatomic and guarantees mutual exclusion at the operation level by associating a short-term lock with the object. TEST is typically an expression comparing (using trans_id's "<" operation) op's newly created trans_id t with other trans_id's embedded in the object's representation. BODY typically computes a result and updates the object's state.

By inheriting from the subatomic class, the implementor can define new classes like atomic_T, and use the operations, in particular those encoded in the when statement, provided by subatomic to implement atomic_T's operations. Since most operations follow the above template, the cleverness required in implementing operations of a new atomic type is in figuring out what the synchronization conditions on atomic_T's operations are and then encoding a test for these conditions in each of the operation's TEST in order to maintain the commit-time order of transactions. ${ }^{1}$ Proving correctness of the implementation focuses on showing that the synchronization conditions permit for only atomic object histories.

Objects defined in a class that inherits from subat omic can also provide comit and abort operations that are called by the system as transactions commit or abort. A user-defined commit typically discards recovery information for the committing transaction, and a user-defined abort typically discards the tentative changes made by the aborting transaction. Intuitively, commit and abort operations in Avalon/C++ are expected to affect liveness, but not safety. For example, delaying a commit or abort operation may delay other transactions (e.g., by failing to release locks) or reduce efficiency (e.g., by failing to discard unneeded recovery information), but it should never cause a transaction to observe an erroneous state. We do not address liveness properties in this paper, though certain ones are clearly of great interest. We would need to rely on the extensive work on temporal logic, e.9., [28], for reasoning about liveness.

\footnotetext{
Ift remains an open research problem to figure out how to derive these synchronization conditions in a systematic way, as one doos in computing weakest pre-conditions.
} 


\section{An Example: A Highly Concurrent FIFO Queue}

In this section, we lllustrate our verification technique by applying it to a highly concurrent atomic FIFO queue implementation. Our implementation is interesting for two reasons. First, it supports more concurrency than commutativity-based concurrency control schemes such as two-phase locking. For example, it permits concurrent enqueuing transactions, even though enqueuing operations do not commute. Second, it supports more concurrency than any locking-based protocol, because it takes advantage of state information. For example, it permits concurrent enqueuing and dequeuing transactions while the queue is non-empty.

We first give the Avalon/C++ implementation of the queve, then define the verification tools needed to prove its correctness, and then give a correctness proof.

\subsection{The Implementation}

As in the implementation of any abstract type, we present first the representation of the abstract type and then the implementations of each of the operations.

\subsubsection{The Representation}

We record information about enq operations in the following struct:

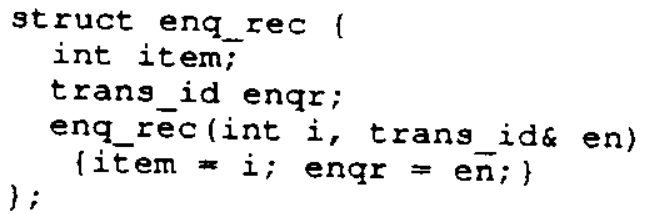

// Item enqueued.

// who enqueued it.

// Constructor.

The item component is the enqueued item. The enqr component is a trans_id generated by the enqueuing transaction. The last component defines a constructor operation for initializing the st ruct.

We record information about deq operations similarly, where the deqr component is a trans_id generated by the dequeuing transaction:

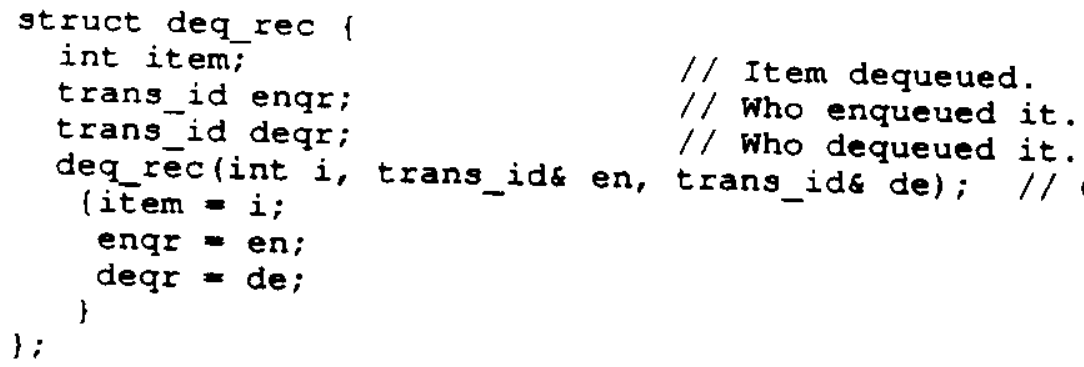

We represent the queve as follows: 


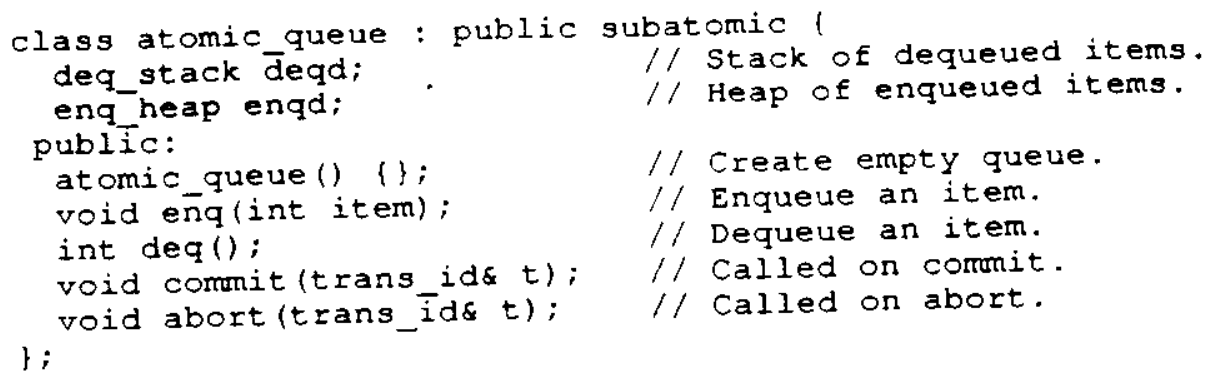

The deqd component is a stack of deq_rec's used to undo aborted deq operations. The enqd component is a partially ordered heap of enq_rec's, ordered by their enqr fields. A partially ordered heap provides operations to enqueue an enq_ rec, to test whether there exists a unique oldest enq_rec, to dequeue it if it exists, and to discard all enq_rec's inserted by (aborted) transactions.

A typical scenario is that when an enq operation occurs, a new trans_id is generated and stored in a new enq_rec, along with the item being enqueued; the enq_rec is inserted in the heap. When a deq operation occurs, a new trans_id is generated and stored in a new deq_rec, along with the information contained in the unique oldest enq_ rec removed from the heap; this deq_rec is pushed on the stack.

\subsubsection{The Operations}

If $B$ is an active transaction, then we say $A$ is committed with respect to $B$ if $A$ is committed, or if $A$ and $B$ are the same transaction. Enq and deq must satisty the following synchronization constraints to ensure atomicity. Transaction A may dequeue an item if (1) the most recent transaction to have executed a deq is committed with respect to $A$, and (2) there exists a unique oldest element in the queue whose enqueuing transaction is committed with respect to $A$. The first condition ensures that $A$ will not have dequeved the wrong item if the earlier dequeuer aborts, and the second condition ensures that there is something for $A$ to dequeue. Similarly. A may enqueve an item if the last item dequeved was enqueued by a transaction committed with respect to $A$.

Given these conditions, here is the code for enq:

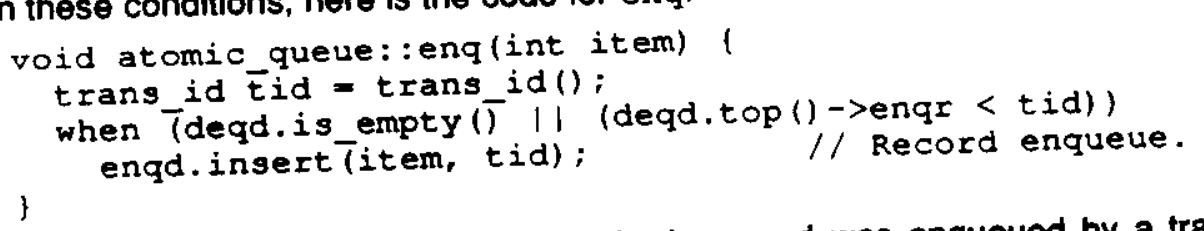

Enq checks whether the Hem most recently dequeued was enqueued by a transaction committed with respect to the caller. If so, the current trans_id and the new item are inserted in enqd. Otherwise, the transaction releases the short-term lock and tries again later (guaranteed by the implementation of the when statement). The somewhat complicated synchronization condition for eng is needed because transactions can perform multiple operations which must be ordered in the sequence in which they were called. (As an aside, the condition is also necessary and sutficient for nested transactions.) Consider the situation depicted in Figure 5-1. $A$ and $B$ are two transactions where $A$ performs two operations. Suppose $A$ is still active. B must wait for $A$ to commit because if $B$ commits at an eartier time than $A$ the 


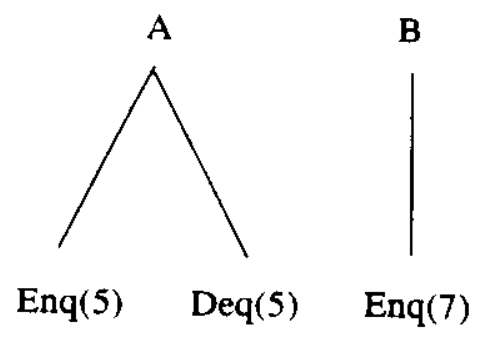

Figure 5-1: An Example of Why an Enquever (B) Must Wait

second operation of $A$ will have dequeued the wrong item (7, not 5, would be at the head of the queue).

Here is the code for deq:

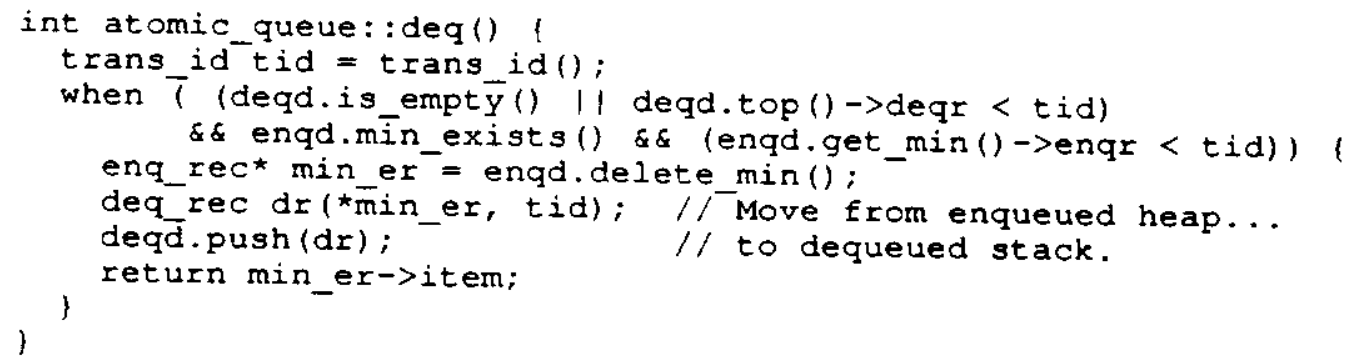

Deq tests whether the most recent dequeuing transaction has committed with respect to the caller, and whether enqd has a unique oldest item. If the transaction that enqueued this Item has committed with respect to the caller, it removes the item from engd and records it in deqd. Otherwise, the caller releases the short-term lock, suspends execution, and tries again later. It is easy to see why a dequeuing transaction $B$ must wait for the dequeuer $A$ of the last dequeued item to be committed with respect to $B$. If $B$ proceeds to dequeue without waiting for $A$ to complete, then it will have dequeued the wrong item if $A$ aborts. Consider the situation in Figure 5-2 where 5 and 7 are the first and second elements in the queve. If $A$ aborts then $B$ should get a 5 .

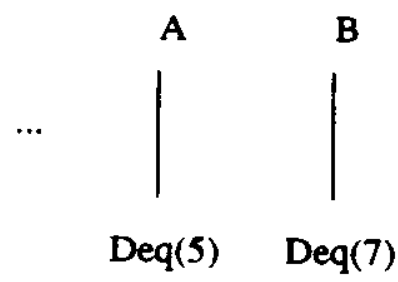

Figure 5-2: An Example of Why a Dequeuer (B) Must Wait

Note that an enquever does not have to wait for the dequever of the last dequeved ifem to commit. Consider the situation in Figure 5-3. Suppose $A$ has committed, but $B$ has not. $C$ can proceed to enqueve a 7 even though $B$ has not yet completed. If $B$ commits, it does not matter whether $t$ commits before or after $C$. B will correctly see 5 at the head of the queve either way and $C$ will correctly place 7 as the new head. If $B$ aborts, then $C$ will correctly place 7 after 5 , which remains at the head of the queve. Thus, $C$ can proceed without waiting for $B$ to complete because there is no way $C$ can be serialized before $A$ and it does not matter in which order $B$ and $C$ are serlallzed. 


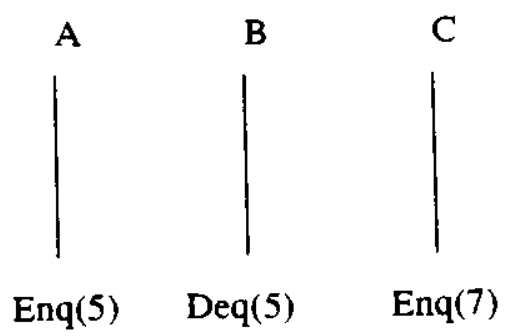

Figure 5-3: An Example of When an Enqueuer (C) Need Not Wait

In addition to the enq and deq operations, the atomic_queue provides comit and abort operations that are applied to the queue as transactions commit or abort. The comit operation looks like:

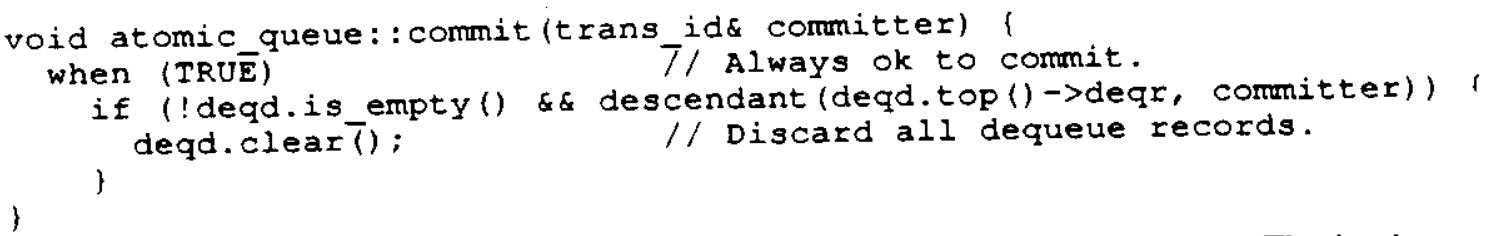

When a transaction commits, it discards deq_rec's no longer needed for recovery. The implementation ensures that all deq_rec's below the top are also superfiuous, and can be discarded. We state this property formally when giving the representation invariant in Section 5.2.1.

The abort operation looks like:

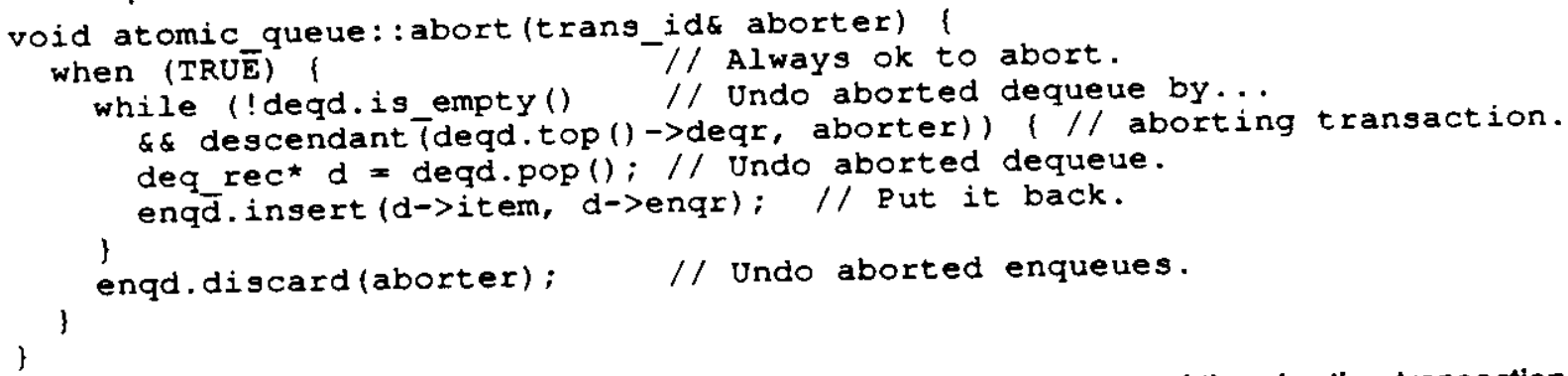

Abort undoes every operation executed by a transaction that is a descendant of the aborting transaction. It interprets deqd as an undo log, popping records for aborted operations, and inserting the items back in enqd heap. Abort then flushes all items enqueved by the aborted transaction and its descendants.

\subsection{Application of Veriflcation Method}

As outlined in Section 3, we need to provide a representation invariant, abstraction function, and sequential specification in order to apply our verification method.

\subsubsection{Representation Invariant}

The queve operations preserve the following representation invariant. For brevity, we assume items in the queve are distinct, an assumption that could easily be relaxed by tagging each item in the queve with a timestamp. For all representation values $r$ :

1. No item is present in both the deqd and enqd components:

$(\forall$ d: deq_rec) $(\forall$ e: enq_rec) $(d \in$ r.deqd $\wedge \in \in$ r.enqd $\Rightarrow$ e.item $\neq$ d.item) 
2. Items are ordered in deqd by their enqueuing and dequeuing trans id's:

$$
\left(\forall \mathrm{d} 1, \mathrm{~d} 2: \text { deq_zec) } \mathrm{d} l<<_{d} \mathrm{~d} 2 \Rightarrow \text { (d1.enqr }<\mathrm{d} 2 \text {.enqr } \wedge \mathrm{d} 1 \text {. deq } r<\mathrm{d} 2 .\right. \text { deqr) }
$$
where $<_{d}$ is the total ordering on deq_rec's imposed by the deqd stack.

3. Any dequeued item must previously have been enqueued:

$$
(\forall \mathrm{d}: \text { deq_rec) } d \in \text { r.deqd } \Rightarrow \text { d.enqr }<\text { d.deq } \text {. }
$$

Thus, given an arbitrary state of the queue representation as in Figure 5-4, where the stack grows upward: The first part of the representation invariant Implles that $x$ (and $y$ ) cannot be in any enq_rec in
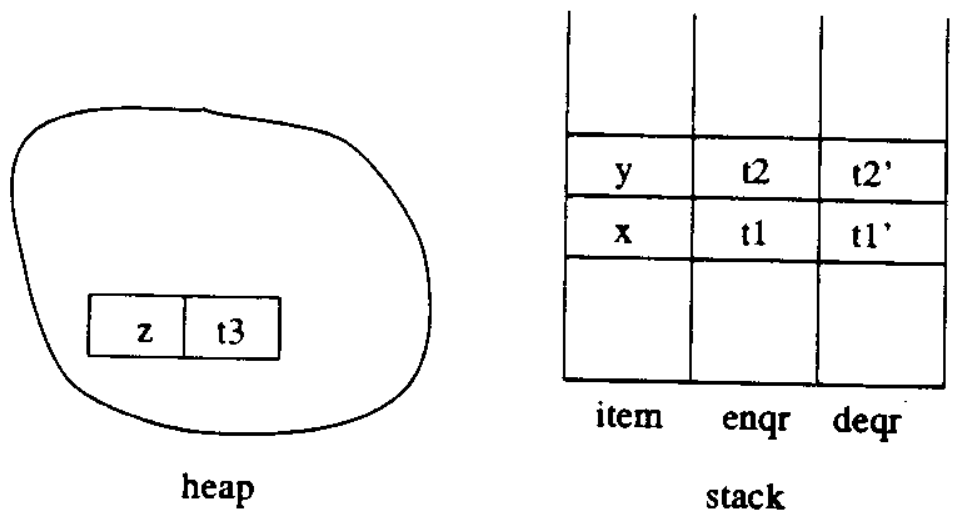

Figure 5-4: An Example Queve Representation State

the heap. The second implies that $t 1<t 2$ and $t 1^{\prime}<t 2$. The third implies that $t 1<t 1^{\prime}($ and $t 2<t 2)$.

Our proof technique requires that we show the representation invariant is preserved across the implementation of each abstract operation. We conjoin it to the pre-and postconditions of each of the operations' specifications.

\subsubsection{Abstraction Function}

Intuitively, the abstract value of the queue is defined in terms of what has been enqueued by committed transactions and may possibly be enqueued by active transactions (what is in the heap) and what has been and may possibly be dequeued (what is on the stack). On-line atomicity requires that we allow for the possibility of active transactions to commit. For each, we pretend that it commits and reflect its effects - tentative enqueues and dequeues saved in the heap and stack-in the image of the abstraction function for a given representation value. When an active transaction actually does commit or the object finally finds out about the transaction's commitment, we know that we have already permitted for its effects to have taken place. Nottce that both commit and abort do not change the abstract view of the queue, but only the representation.

To define the abstraction function, we need some auxiliary definitions. Let $Q$ be a sequential queue history (not necessarily legal). Define the auxiliary functions $E N Q(Q)$ and $D E Q(Q)$ to yield the sequences of items enqueued and dequeued in $O$ :

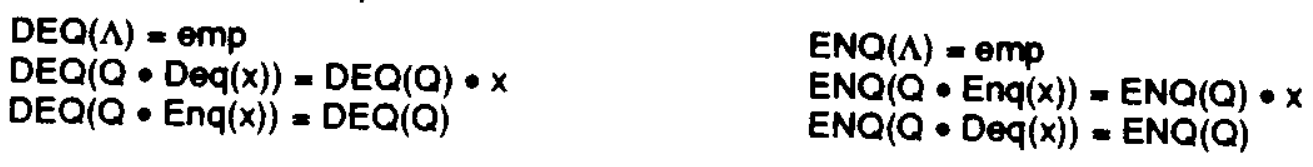


For an operation $p$ that is neither an $\operatorname{Enq}(x)$ nor $\operatorname{Deq}(x)$ :

$$
\mathrm{DEQ}(Q \cdot p))=\mathrm{DEQ}(\mathrm{Q}) \quad \mathrm{ENQ}(\mathrm{Q} \cdot p)=\mathrm{ENQ}(\mathrm{Q})
$$

Here, "Enq(x)" is shorthand for an Enq operation performed by some transaction $A$, "q Enq(x) $A$ $q \operatorname{Ok}() A^{\prime}$ and "Deq(x)" is shorthand for "q $\operatorname{Deq}() A \cdot q \operatorname{Ok}(x) A . "$ Here, "emp" denotes the empty sequence of items.

Let $T$ be the universe of all (unique) trans_id's. $P \subseteq T$ is a prefix set if, $\forall t, t^{\prime} \in T$, if $t \in P \wedge t^{\prime}<t$, then $t^{\prime} \in P$. (The lemma below is independent of the queve example.)

Lemma 10: If $H$ is an on-line atomic history for a set of trans id's and $S$ is a serialization of $H$, then the trans_id's whose creation operations appear in $S$ form a prefix set.

Given a representation value $r$ and a prefix set of trans_id's, we define the auxiliary function OPS $(r, P)$ to yield the partially ordered set of operations tagged by trans_id's in $P$. OPS $r, P)$ for the queve example is equal to the following set:

$$
\begin{gathered}
\{\text { Enq }(x) \mid(\exists \text { e:enq_rec } \in \text { r.enqd) e.item }=x \wedge \text { e.enqr } \in P \vee \\
\text { (ヨ d:deq_rec } \in \text { r.deqd) d.item }=x \wedge \text { d.enq } x \in P\} U \\
\{\text { Deq(y) }(\exists \text { d:deq_rec } \in \text { r.deqd }) \text { d.item }=y \wedge \text { d.deq } x \in P\}
\end{gathered}
$$

Each operation is tagged with a trans_id (e.enqr, d.enqr, or d.deqr). These trans_id's induce a partial order on the elements of $O P S(r, P)$.

We define Present' to take a representation value $r$ and a prefix set $P$, from which we can define $O P S(r$, $P$ ). The value of Present' $(r, P)$ is a set of sequences of (queve) operations where each sequence has the same elements as in OPS $(r, P)$ in a (total) order that extends the partial order of OPS $(r, P)$.

Present $(r, P)=\{Q \mid$ elements $(O P S(r, P))=\operatorname{elements}(Q) \wedge \operatorname{order}(O P S(r, P)) \subseteq \operatorname{order}(Q)\}$

Present $(r)$ is defined as the union of Present' $(r, P)$ over all prefix sets $P \subseteq T$ :

$$
\text { Present }(r)=\underset{P}{\cup} \text { Present' }(r, P)
$$

From the representation of the queue, we can see that a representation value $r$ can keep track of only those items enqueued but not yet dequeued by committed transactions. In order to apply our verification method $(\operatorname{Ser}(H) \subseteq A(r))$, we must consider all possible past histories that could have gotten the representation to its present state. So, we use Past to generate an infinite set of finite prefixes for a queue history:

Past $=\{Q \mid Q$ is a sequential queve history $\wedge D E Q(Q)=E N Q(Q)\}$

Finally, we define $A(r)$ as follows:

$$
A(r)=\left\{Q \mid \exists Q_{1} \in \text { Past, } \exists Q_{2} \in \text { Present(r). } Q=Q_{1} \cdot Q_{2}\right\}
$$

Note that $A(r)$ typically includes more histories than Ser(H), the set of serializations of $H$.

Defining abstraction functions for other implementations of atomic queues and for implementations of other data types follow the same pattern. Since operations are typically tagged by a trans_id, we 
gather up these trans_id's into a prefix set $P$, define a type-specific OPS $(r, P)$ (the elements are just individual instances of the operations provided by the type), define a Past function that lets us generate all possible legal prefixes ${ }^{2}$, and finally a Present'( $r, P$ ) function that lets us tum a set of partially ordered operations into a set of totally ordered ones, i.e., sequences of operations.

\subsubsection{Type-Specific Correctness Condition}

The sequential queue specification in Figures 2-1 and 2-2 implicitly defines the set of legal sequential queue histories, and thus, the set of legal abstract queue values. Our verification method requires that we reason about serializations of a given queue history, e.g., showing that every history in $A(r)$ is legal. Recall we introduced serializations to capture the "pessimistic" property of on-line atomicity where a serialization of a history $H$ is a sequential equivalent version of $H$ with appended commit events for active transactions. Since an object may actually learn of the commitment of transactlons in an order different from their actual commit times, we need a way to recognize when it is legal to insert an operation "In the middle" of a legal sequential history. The following lemma about sequential queue histories captures this notion.

Lemma 11: Let $Q=Q_{1} \bullet Q_{2}$ be a legal sequential queve history, and let $p$ be a queve operation. The sequential history $Q^{\prime}=Q_{1} \bullet p \cdot Q_{2}$ is legal if $D E Q\left(Q^{\prime}\right)$ is a prefix of $E N Q\left(Q^{\prime}\right)$.

This lemma captures the "prefix" property of a queue's behavior. It indirectly characterizes the conditions under which queve operations may execute concurrently; an analogous lemma would be needed for any other data type to be verified.

To illustrate why we need to possibly reorder operations in a history, consider the following history:

$\begin{array}{lll}\text { q } & \operatorname{Enq}(5) & \text { A } \\ \text { q } \text { Ok() } & \text { A } \\ \text { q } & \text { Enq(7) } & \text { A } \\ \text { q } \text { Ok() } & \text { A } \\ \text { q } \text { Deq() } & \text { A } \\ \text { q Ok(5) } & \text { A } \\ \text { q Commit(1:00) } & \text { A } \\ \text { q Enq(1) } & \text { B } \\ \text { q Ok() } & \text { B } \\ \text { q } \text { Deq() } & \text { C } \\ \text { q Ok(7) } & \text { C } \\ \text { q Enq(8) } & \text { C } \\ \text { q Ok() } & \text { C }\end{array}$

$q$ must permit for $B$ and $C$ to commit, and in either order. Though the sequence of operations that $q$ sees is:

$$
\operatorname{Enq}(5) \cdot \operatorname{Enq}(7) \cdot \operatorname{Deq}(5) \cdot \operatorname{Enq}(1) \cdot \operatorname{Deq}(7) \cdot \operatorname{Enq}(8)
$$

since $C$ may commit before $B$ it needs to permit for this sequence as well:

$$
\operatorname{Enq}(5) \cdot \operatorname{Enq}(7) \cdot \operatorname{Deq}(5) \cdot \operatorname{Deq}(7) \cdot \operatorname{Enq}(8) \cdot \operatorname{Enq}(1)
$$

where C's operations are inserted before Bs. We need to check that both sequences are legal; and

\footnotetext{
${ }^{2}$ Here again, an object's sequential specification, i.e., its data type semantics, determines how to define Past.
} 
indeed, $\langle 5,7\rangle$ is a prefix of both $\langle 5,7,1,8\rangle$ and $\langle 5,7,8,1\rangle$.

\subsection{Verifying the Implementation}

We verify the queue implementation by showing inductively that every sequential history in $\operatorname{Ser}(H)$ lies in $A(r)$ and that every sequential history in $A(r)$ is legal. First we give an informal summary of our arguments and then the more formal proofs for the Enq and Deq operations.

\subsubsection{Proof Sketch}

Suppose the object completes an operation Enq(x) with trans_id $t$, carrying the accepted history $H$ to $H^{\prime}$, and the representation $r$ to $r^{\prime}$. It is immediate from Lemma 10 that $\operatorname{Ser}\left(H^{\prime}\right) \subseteq A\left(r^{\prime}\right)$. To show that every history in $A\left(r^{\prime}\right)$ is legal, let $Q^{\prime} \in A\left(r^{\prime}\right)$. If $Q^{\prime}$ fails to satisty the prefix property of Lemma 11, there must exist $y$ in $D E Q\left(Q^{\prime}\right)$ such that $x$ precedes $y$ in ENQ(Q'), implying that the Enq of $x$ is serlalized before the Enq of $y$. Let $t^{\prime}$ be the enqueuing trans_id for the item at the top of the deqd stack, and let $t$ " be the enqueuing trans_id for $y$. The when condition for Enq ensures that $t^{\prime}<t$, and the representation invariant ensures that $t^{\prime \prime} \leq t^{\prime}$, hence that $t^{\prime \prime}<t$, which is impossible if the Enq of $x$ is serialized first.

Similarly, suppose the object completes an operation Deq $(x)$ with trans_id $t$, carrying the representation $r$ to $r$. Let $Q=Q_{1} \cdot Q_{2} \in A(r)$ and $Q^{\prime}=Q_{1} \bullet \operatorname{Deq}(x) \cdot Q_{2} \in A\left(r^{\prime}\right)$. The representation invariant and the first conjunct of the when condition for Deq ensure that $x$ is not an element of $D E Q(Q)$, and the second conjunct then ensures that $x$ is the first element of $E N Q(Q)-D E Q(Q)$. Together, they imply that $D E Q\left(Q^{\prime}\right)=D E Q(Q) \bullet x$ is a prefix of $E N Q\left(Q^{\prime}\right)=E N Q(Q)$, hence that $Q$ is legal by Lemma 11 .

If a Commit or Abort event carries the accepted history $H$ to $H^{\prime}$, and the corresponding comenit or abort operation carries $r$ to $r^{\prime}$, we must show that (1) $A\left(r^{\prime}\right) \subseteq A(r)$, and $(2)$ that no history in $A(r)-A\left(r^{\prime}\right)$ is in Ser $\left(H^{\prime}\right)$. Property 1 ensures that every sequential history in $A\left(r^{\prime}\right)$ is legal, and Property 2 ensures that no valid serializations are "thrown away." For Commit, we check that every discarded history is missing an operation of a committed transaction, and for Abort, we check that every discarded history includes an operation of an aborted transaction; either condition ensures that the discarded history is not an element of $\operatorname{Ser}\left(H^{\prime}\right)$.

Naturally, this verification relies on properties of sequential queues. To verify an implementation of another data type, one would have to rely on a different set of properties, but the arguments would follow a similar pattem. The basic synchronization conditions are captured by a type-specific analog to Lemma 11. characterizing the conditions under which an operation can be inserted in the middle of a sequential history. The representation invariant and abstraction function define how the set of possible serializations is encoded in the representation, and an inductive argument is used to show that no operation, commit, or abort event can violate atomicity. 


\subsubsection{Formal Proof for Enqueue and Dequeue}

In this section we will use induction to show the prefix property of Lemma 11. More specifically, if the prefix property holds of all serializations $h \in A(r)$ at the invocation of the enqueue or dequeue operation, it holds of all serializations $h^{\prime} \in A\left(r^{\prime}\right)$ at the point of return. In the following, for $H \in A(r), H^{\prime} \in A\left(r^{\prime}\right)$, let $H=$ $H_{1} \bullet H_{2}$ and $H^{\prime}=H_{1} \bullet o p \bullet H_{2}$ such that $\forall p \in H_{1} \neg\left(\right.$ t id $<$ trans_id(p)) $\wedge \forall p \in H_{2} \neg($ trans_id(p) < tid), where op is the enqueve or dequeue operation (as the case may be) with trans_id tid, and trans_id $(p)$ is the trans_id of operation $p$.

\section{Enqueuse}

We decorate the enq operation with two assertions, one after the when condition, and one at the point of return.

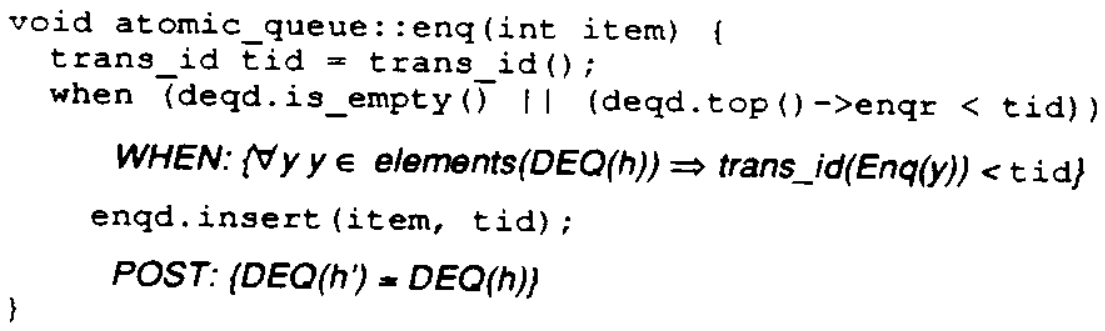

Proof: Case 1: The queve is empty. Trivial since the antecedent of WHEN is false.

Case 2: The queue is nonempty. Then let $y$ be an item dequeued in $H$, which implles that the trans_id of the enqueue operation of $y$ is ordered betore tid by the WHEN assertion. The enqueue operation must be in $H_{1}$ since (1) the trans_id's of all enqueve operations of dequeued items are all ordered before that of deqd.top().enqr (by the representation invariant), which is ordered before tid (by the when condition); and (2) tid is not ordered before any operation in $H_{1}$ (by the definition of $H=H_{1} \bullet H_{2}$ ). Since the enqueue operations of all dequeued items are in $H_{1}$,

$\operatorname{DEQ}(H)$ prefix $\operatorname{ENQ}\left(H_{1}\right)$

At the point of return, let $\theta=E n q(x)$. From POST we have that:

$\operatorname{DEQ}(H)=\operatorname{DEQ}(H)$, which by $(*)$

$\Rightarrow \mathrm{DEQ}(H)$ prefix $\operatorname{ENQ}\left(H_{1}\right)$

$\Rightarrow \mathrm{DEQ}(H)$ prefix ENQ $\left(H_{1} \bullet \theta \bullet H_{2}\right)$

$\Rightarrow \mathrm{DEQ}(H)$ prefix of $\mathrm{ENQ}(H)$. 
Dequeue

Here is the annotated deq operation:

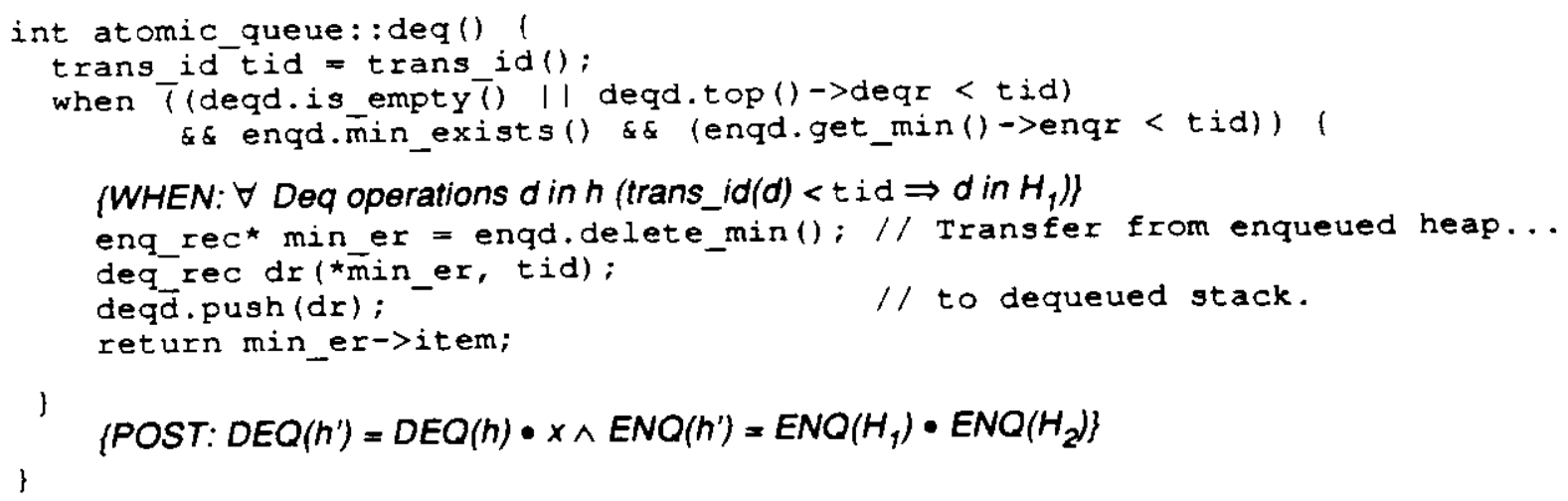

and the proof:

Proof: From the first conjunct of the when condition and the second clause of the representation invariant, we know that $\mathrm{DEQ}(H)=\mathrm{DEQ}\left(H_{1}\right)$. The second conjunct implies that there exists some $x=$ first(ENQ $(H)-D E Q(H))$, the first item in the sequence of enqueued items that have not yet been dequeued. The third conjunct implles that this them, $x$, is in $H_{1}$. Thus, by properties on sequences, there exists some $x=f i r s t\left(E N Q\left(H_{1}\right)-D E Q\left(H_{1}\right)\right)$.

At the point of return, let $d=\operatorname{Deq}(x)$. POST implies that

$$
\begin{aligned}
& \operatorname{DEQ}\left(H _ { 1 } \cdot \text { d) prefix } \operatorname { E N Q } \left(H_{1} \bullet\right.\right. \text { d) } \\
& \Rightarrow \operatorname{DEQ}(H) \text { prefix } \operatorname{ENQ}\left(H_{1} \cdot d\right) \\
& \Rightarrow \operatorname{DEQ}(H) \text { prefix } \operatorname{ENQ}\left(H_{1} \bullet d \cdot H_{2}\right) \\
& \Rightarrow \operatorname{DEQ}(H) \text { prefix } \operatorname{ENQ}(H) .
\end{aligned}
$$

\section{Discussion and Related Work}

\subsection{Hybrid Atomicity Revislted}

Atomicity has long been recognized as a basic correctness property within the database community [3]. More recently, several research projects have chosen atomicity as a useful foundation for generalpurpose distributed systems, including Avalon [18], Argus [24], Aeolus [39], Camelot [34], EXODUS [11] and Arjuna [9]. EXODUS and Arjuna, like Avalon, extend $C_{++}$to support recoverability, but neither gives programmers fine control over serializability. Of all these projects only Avalon and Argus provide linguistic support for programmers to design and implement user-defined atomic data types, which Weihl and Liskov argue is necessary for building large, realistic systems [37].

One way to ensure atomicity of a set of concurtent transactions is to associate read and witte locks wth each object and to use a strict two-phase locking protocol to ensure serializability [10]. A transaction $A$ obtains a read lock on an object $x$ if $A$ needs only to observe $x$ 's value. It obtains a write lock if it needs to update $x$ 's value. Each transaction first acquires all the locks it needs, then performs its operations on all objects for which it has obtained the appropriate locks, and then when it commits or aborts, releases all of its locks. Locks are held for the duration of a transaction, not individual operations; thus, two transactions 
that need to perform updates on the same object cannot proceed concurrently.

The two-phase read-write locking protocol is known to guarantee atomicity. However, since operations are naively divided into readers and writers, the amount of concurrency that can be obtained is restricted because the type semantics of objects are ignored. For example, consider the following two transactions that each perform two enqueve operations:

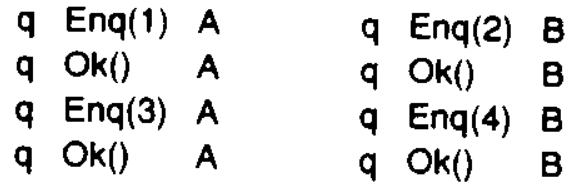

Following a read-write locking protocol would prevent $A$ and $B$ from executing concurrently. Suppose $A$ has the write lock on $q$, then $B$ would not be able to obtain it, and hence has to wait for $A$ to commit or abort before proceeding. Assuming both $A$ and $B$ commit, the only two permissible histories would both be sequential, where either all of $A$ 's operations precede all of Bs or vice versa, i.e., A's and Bs operations would not be interleaved. However, it should be possible to permit for the following history in which $A$ and $B$ are executing concurrently:

$\begin{array}{lll}q & \operatorname{Enq}(1) & A \\ q & \text { Ok() } & \text { A } \\ q & \operatorname{Enq(2)} & \text { B } \\ \text { q } & \text { Ok() } & \text { B } \\ q & \operatorname{Enq(3)} & \text { A } \\ q & \text { Ok() } & \text { A } \\ \text { q } & \text { Enq(4) } & \text { B } \\ \text { q } & \text { Ok() } & \text { B }\end{array}$

Our correctness condition (hybrid atomicity) certainly permits this history since any extension of it with appended commit events for $A$ and/or $B$ is serializable.

Moreover, in the case when the queue is non-empty, we can permit a dequeuing transaction to proceed concurrently with an enqueuing one. Consider this example, which is a variation of the example drawn in Figure 5-3:

$\begin{array}{lll}\text { q } & \text { Enq(1) } & \text { A } \\ \text { q } \text { Ok() } & \text { A } \\ \text { q Enq(3) } & \text { A } \\ \text { q } \text { Ok() } & \text { A } \\ \text { q } \text { Commit(1:00) } & \text { A } \\ \text { q Enq(2) } & \text { B } \\ \text { q Deq() } & \text { C } \\ \text { q Ok(1) } & \text { C } \\ \text { q Ok() } & \text { B } \\ \text { q Enq(4) } & \text { B } \\ \text { q Ok() } & \text { B }\end{array}$

The queve can permit $C$ to perform a Deq operation and even retum an element to $C$ because it knows that $A$ has committed and thus it knows what its first element is. Whether $B$ commits or not, $C$ still 
receives the correct element. Were two-phase read-write locking used, $B$ and $C$ would not be allowed to proceed concurrently because the Enq and Deq operations would both be classified as writers.

The use of commit-time serialization distinguishes Avalon from other transaction-based languages and systems, which are typically based on some form of strict two-phase locking. We chose to support commit-time serialization because it permits more concurrency than two-phase locking [36], as well as better availability for replicated data [17]. Because commit-time serialization is compatible with strict two-phase locking, applications that use locking can still be implemented in Avalon/C+t. In fact, we optimize for this more traditional case: As an alternative way to build atomic data types, programmers can inherit from another built-in Avalon/ $\mathrm{C}_{+}+$called atomic, which provides access to read and write locks.

To summarize the results of this discussion and that in Section 2, the Venn diagram in Figure 6-1 shows the relationship between atomic, hybrid atomic, and "two-phase-locking" atomic histories. Every "twophase locking" history is hybrid atomic, but not conversely; every hybrid atomic history is atomic, but not conversely. The key point of this section is that hybrid atomicity provides more concurrency than "twophase locking." The key point with respect to this paper, however, is that hybrid atomity is local, whereas atomicity is not.

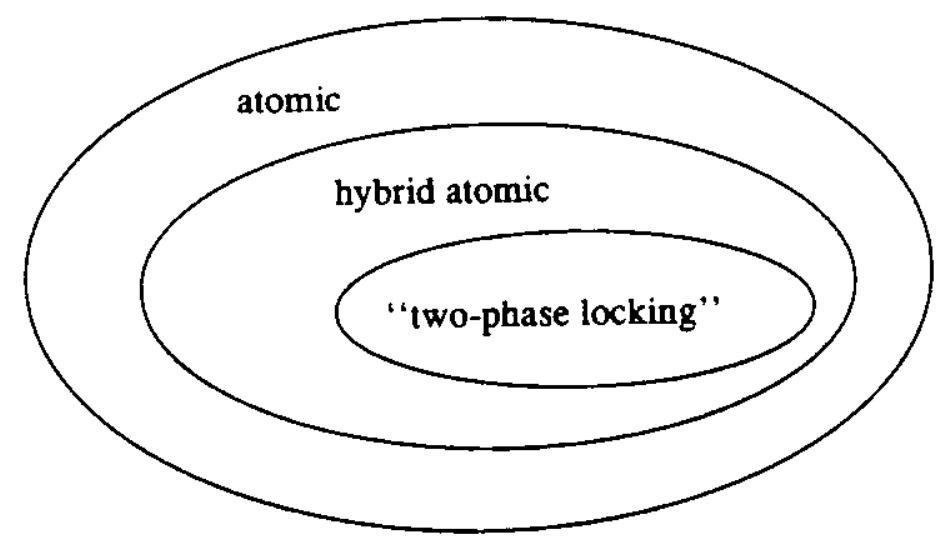

Figure 6-1: Relationships Among Atomicity Properties

\subsection{Abstraction Functions Revisited}

The main contribution of this paper is the verification method used for showing the correctness of the implementation of an atomic data type. This method hinges on defining an abstraction function between a low-level view of an object and an abstract view. In the sequential domain, the signature of the abstraction function is:

\section{A: $R e p \rightarrow$ Abs}

Because of the on-line property of our correctness condition, in particular the inherent nondeterminism, we need to map to a set of values. A first attempt at extending the abstraction function would be to extend the range as follows:

A: $R e p \rightarrow 2^{A b s}$ 
This extension is similar to Lynch and Tuttle's use of multi-valued possibilities mappings, where each "concrete state" maps to a set of "abstract states" [27]. Lynch and her colleagues have used possibilities mappings to prove a wide range of distributed algorithms correct, including transaction-based locking and timestamp protocols. This abstraction function extension is also similar to what Herlihy and Wing needed to use in order to prove the correctness of linearizable objects [19], again because of the inherent nondeterminism in the definition of the correctness condition.

However, even mapping to the powerset of abstract values is insufficient for the on-line hybrid atomic correctness condition we require. We need to keep track of sequences of operations because we need to permit for reordering of operations. In fact, we need to be able to insert not just single operations into the middle of a history, but sequences of operations since a transaction may perform more than one operation. Hence, we finally extend the abstraction function's range to be:

$$
\text { A: } R e p \rightarrow 2^{O P \cdot}
$$

In either extension, the standard trick of using auxiliary variables (Abadi and Lamport classify these into history and prophecy variables [1]) would also work. These varlables can be included in the domain of the abstraction function (encoded as part of the representation state) and used (1) to keep track of the set of possible abstract values; (2) to log the history of abstract operations performed on the object so far; and (3) to keep track of implicit global data like the trans_id tree. Hence our abstraction functions can be turned into Abadi and Lamport's refinement mappings, where the extended domain of the representation state maps to a single abstract state.

In our initial approach to veritication we tried to stick to a purely axiomatic approach in our verification method where we relied on Hoare-like axioms to reason about program statements, invariant assertions to reason about local state changes and global invariants, and auxillary variables to record the states (e.g., program counters) of concurrent processes. In the transactional domain, however, an atomic object's state must be given by a set of possible serializations, and each new operation (or sequence of operations) is inserted somewhere "in the middle" of certain serializations. This distinction between physical and logical ordering is easily expressed in terms of reordering histories, but seems awkward to express axiomatically, i.e., using assertions expressed in terms of program text alone. Though the proots given in this paper fall short of a pure syntax-directed verification, they could be completely axiomatized by encoding the set of serializations as auxiliary data. Even so, we have found that the resulting invariant assertions are syntactically intimidating and the proots unintuitive and unnatural.

\subsection{Other Models for Transactions}

Best and Randell [4], and Lynch and Merritt [26] have proposed formal models for transactions and atomic objects. Best and Randell use occurrence graphs to define the notion of atomicity, to characterize interference freedom, and to model error recovery. Their model does not exploit the semantics of data, focusing instead on event dependencies. Lynch and Merritt model nested transactions and atomic objects in terms of $1 / O$ automata, which have been used to prove correctness of general algorithms for synchronization and recovery $[12,25]$. None of these models were intended for reasoning about 
individual programs. Moreover, none are suitable for reasoning about high-level programming language constructs that include support for user-defined abstract data types.

\section{Current and Future Work}

We have applied our verification method to a directory atomic data type, whose behavior is much more complex than a queue's. A directory stores key-item pairs and provides operations to insert, remove, alter, and lookup items given a key. Synchronization is done per key so transactions operating on different keys can execute concurrently. Moreover, we use an operation's arguments and results to permit, in some cases, operations on the same key to proceed concurrently. For example, an "unsuccessful" insertion operation, i.e., Ins( $k, x) / O k$ (false), does not modity $k$ 's binding, so it does not conflict with a "successful" lookup operation, i.e., Lookup(k)/OK(y). Our proof of correctness relies on inductively showing a type-specific correctness condition, analogous to the "prefix" property for the queve. Informally stated, this condition says that it is legal to pertorm a successful remove, alter, lookup or unsuccessful lookup or insert on a key $k$ as long as a key-item pair has already been successfully inserted for $k$ and not yet successtully removed. This condition should hint to the reader that, in general, we need to keep track of the exact operations (including their arguments and results) and the order they have occurred already in a history to know which permutations are legal. We implemented the directory example in Avalon/C++.

In line with our philosophy of performing syntax-directed verification, we have used machine aids to verify the queve example. In particular, we used the Larch Shared Language to specify completely the queue representation, the set of queue abstract values (in terms of sequences of queue operations), the representation invariant, and the abstraction function. We used the Larch Prover [13] to prove the representation invariant holds of the representation and to perform the inductive reasoning we carried out in our verification method. We describe this work in more detail in [15].

Our work on specifying and verifying atomic data types and more recently, our work on using machine aids, has led us to explore extensions to our specification language. Two kinds of extensions seem necessary. First, we need a way to specify precisely and formally the synchronization conditions placed on each operation of an atomic object. We propose using a when clause analogous to the when statement found in Avalon/C++ or the WHEN assertion found in our proots. Birrell et al. use informally a when clause in the Larch interface specification of Modula/2+'s synchronization primitives [5] and Lerner uses it to specify the queue example [23]. Second, we would like to extend the assertion language of the Larch interface specifications. The Larch Shared Language and the input language of the Larch Prover are both restricted to a subset of first-order logic. The assertions we write, however, in both the PREMHEN/POST conditions in our proots and the requires/when/ensures clauses in our Larch interfaces, refer to operations, histories, sets of histories, and transactions directly, thereby requiring a richer and more expressive language than that which either the Larch Shared Language or the Larch Prover supports. 


\section{Acknowledgments}

I would like to thank Maurice Herlihy specifically for his close collaboration on the Avalon/C++ design and implementation, for his ideas about verifying atomicity, and for co-authoring a paper that presents a preliminary version of the some of the ideas in this paper. I thank Bill Weihl for the original definitions of his model of transactions and atomicity properties.

I thank the participants of the 1989 REX Workshop on Stepwise Refinement of Distributed Systems: Models, Formalism, Correctness that took place in Plasmolen, The Netherlands. I especially thank explaining this material.

Finally, I thank the following members of the Avalon Project for reading drafts of this paper: Chun Gong, David Detlefs, Karen Kietzke, Linda Leibengood, Rick Lerner, and Scott Nettles. Gong and Dave have been particularly helpful with some of the technical points. Dave and Karen have been instrumental in turning the design of Avalon/C++ into a working implementation.

This research was sponsored by the Defense Advanced Research Projects Agency (DOD), ARPA Order No. 4976, monitored by the Air Force Avionics Laboratory Under Contract F33615-87-C-1499. Additional support was provided in part by the National Science Foundation under grant CCR-8620027. The views and conclusions contained in this document are those of the author and should not be interpreted as representing the official policies, either expressed or implied, of the Defense Advanced Research Projects

\section{References}

[1] M. Abadi and L. Lamport.

The Existence of Refinement Mappings.

Technical Report 29, DEC Systems Research Center, August, 1988.

[2] K.R. Apt, N. Francez, and W.P. DeRoever. A Proof System for Communicating Sequential Processes.

[3] P.A. Bernstein and N. Goodman. A survey of techniques for synchronization and recovery in decentralized computer systems.

[4] E. Best and B. Randell. A Formal Model of Atomicity in Asynchronous Systems.

[5] A. Birrell, J. Guttag, J. Horning, R. Levin. Synchronization Primitives for a Multiprocessor: A Formal Specification. In Proceedings of the Eleventh ACM Symposium on Operating Systems ACM/SIGOPS, 1987.

[6] D. Bjorner and C.G. Jones (Eds.).

Lecture Notes in Computer Science. Volume 61: The Vienna Development Method: the
Meta-language. Springer-Verlag, Berlin-Heidelberg-New York, 1978. 
[7] R.M. Burstall and J.A. Goguen.

An Informal Introduction to Specifications Using CLEAR.

In Boyer and Moore (editors), The Correctness Problem in Computer Science. Academic Press, 1981.

[8] D. L. Detlets, M. P. Herlihy, and J. M. Wing.

Inheritance of Synchronization and Recovery Properties in Avalon/C++.

IEEE Computer :57-69, December, 1988.

[9] G. Dixon and S.K. Shrivastava.

Exploiting Type Inheritance Facilities to Implement Recoverability in Object Based Systems.

In Proceedings of the 6th Symposium in Reliability in Distributed Software and Database Systems. March, 1987.

[10] K.P. Eswaran, J.N. Gray, R.A. Lorie, and I.L. Traiger.

The Notion of Consistency and Predicate Locks in a Database System.

Communications ACM 19(11):624-633, November, 1976.

[11] J.E. Richardson and M.J. Carey.

Programming Constructs for Database System Implementation in EXODUS.

In ACM SIGMOD 1987 Annual Conference, pages 208-219. May, 1987.

[12] M.P. Herlihy, N.A. Lynch, M. Merritt, and W.E. Weihl.

On the correctness of orphan elimination algorithms.

In 17th Symposium on Fault-Tolerant Computer Systems. July, 1987.

Abbreviated version of MIT/LCS/TM-329.

[13] S.J. Garland and J.V. Guttag.

Inductive Methods for Reasoning about Abstract Data Types.

In Proceedings of the 15th Symposium on Principles of Programming Languages, pages 219-228.

January, 1988.

[14] J.A. Goguen and J.J. Tardo.

An Introduction to OBJ: A Language for Writing and Testing Formal Algebraic Program

Specifications.

In Proceedings of the Conference on Specifications of Reliable Software, pages 170-189. Boston, MA, 1979.

[15] C. Gong and J.M. Wing.

Machine-Assisted Proofs of Atomicity.

1989.

in preparation.

[16] J.V. Guttag, J.J. Horning, and J.M. Wing.

The Larch Family of Specification Languages.

IEEE Software 2(5):24-36, September, 1985.

[17] M.P. Herlihy.

A quorum-consensus replication method for abstract data types.

ACM Transactions on Computer Systems 4(1), February, 1986.

[18] M.P. Herlihy and J.M. Wing.

Avalon: Language Support for Reliable Distributed Systems.

In The Seventeenth International Symposium on Fault-Tolerant Computing, pages 89-94. July, 1987.

Also available as CMU-CS-TR-86-167.

[19] M.P. Herlihy and J.M. Wing.

Axioms for concurrent objects.

In Fourteenth ACM Symposium on Principles of Programming Languages, pages 13-26. January, 1987. 
[20] C.A.R. Hoare.

Proof of Correctness of Data Representations.

Acta Informatica 1(1):271-281, 1972.

[21] L. Lamport.

Time, clocks, and the ordering of events in a distributed system.

Communications of the ACM 21(7):558-565, July, 1978.

[22] L. Lamport.

Specifying Concurrent Program Modules.

ACM Transactions on Programming Languages and Systems 5(2):190-222, April, 1983.

[23] R.A. Lerner.

Specifying Concurrent Programs.

1989.

Thesis Proposal.

[24] B.H. Liskov, and R. Scheifler.

Guardians and actions: linguistic support for robust, distributed programs.

Transactions on Programming Languages and Systems 5(3):381-404, July, 1983.

[25] N. Lynch.

A Concurrency Control For Resilient Nested Transactions.

Technical Report MIT/LCS/TR-285, Laboratory for Computer Science, 1985.

[26] N. Lynch and M. Merritt.

Introduction to the Theory of Nested Transactions.

In Proceedings of the International Conference on Database Theory. Rome, Italy, September, 1986.

Sponsored by EATCS and IEEE.

[27] N. Lynch and M. Tuttle.

Hierarchical Correctness Proofs for Distributed Algorithms.

Technical Report MIT/LCS/TR-387, Laboratory for Cumputer Science, 1987, 1987.

[28] Z. Manna and A. Pnueli.

Verification of concurrent Programs, Part l: The Temporal Framework.

Technical Report STAN-CS-81-836, Dept. of Computer Science, Stanford University, June, 1981.

[29] J.E.B. Moss.

Nested Transactions: An Approach to Reliable Distributed Computing.

Technical Report MIT/LCS/TR-260, Laboratory for Computer Science, April, 1981.

[30] R. Nakajima, M. Honda, and H. Nakahara.

Hierarchical Program Specification and Verification-- A Many-sorted Logical Approach.

Acta Informatica 14:135-155, 1980.

[31] S. Owicki and D. Gries.

Verifying Properties of Parallel Programs: An Axiomatic Approach.

Communications of the ACM 19(5):279-285, May, 1976.

[32] C.H. Papadimitriou.

The serializability of concurrent database updates.

Journal of the ACM 26(4):631-653, October, 1979.

[33] D.P. Reed.

Implementing atomic actions on decentralized data.

ACM Transactions on Computer Systems 1(1):3-23, February, 1983. 
[34] A.Z. Spector, J.J. Bloch, D.S. Daniels, R.P. Draves, D. Duchamp, J.L. Eppinger, S.G. Menees, D.S. Thompson.

The Camelot Project.

Database Engineering 9(4), December, 1986.

Also available as Technical Report CMU-CS-86-166, Carnegie Mellon University, November 1986.

[35] B. Stroustrup.

The $C_{++}$Programming Language.

Addison Wesley, 1986.

[36] W.E. Weihl.

Specification and implementation of atomic data types.

Technical Report TR-314, MIT Laboratory for Computer Science, March, 1984.

[37] W.E. Weihl, and B.H. Liskov.

Implementation of resilient, atomic data types.

ACM Transactions on Programming Languages and Systems 7(2):244-270, April, 1985.

[38] W.E. Weihl.

Local Atomicity Properties: Modular Concurrency Control for Abstract Data Types.

Transactions on Programming Languages and Systems 11(2):249-283, April, 1989.

[39] C.T. Wilkes and R.J. LeBlanc.

Rationale for the design of Aeolus: a systems programming language for an action/object system. Technical Report GIT-ICS-86/12, Georgia Inst. of Tech. School of Information and Computer Science, Dec, 1986. 